> «Une pandémie silencieuse: dans le monde entier, l'industrie chimique est en train de détériorer le développement cérébral des enfants». C'est ainsi qu'a été relayée dans la grande presse et les mouvements écologistes une importante revue publiée dans le Lancet et rédigée par deux donneurs d'alerte appartenant à l'école de santé publique de Harvard ('́tats-Unis) et travaillant depuis des décennies sur les dangers de l'environnement. Cet article faisait suite à deux études, l'une réalisée en Amérique du Nord par la CCE (commission de coopération environnementale), et l'autre en Europe, intitulée DEVNERTOX et financée par la $C \varepsilon$ à hauteur de 2,4 millions d'euros. Ces travaux soulignent l'inquiétude des organismes de santé publique vis-à-vis de ce risque important. C'est pourquoi, après avoir abordé, dans le numéro de mai 2007 de Médecine/ Sciences, les effets de la pollution atmosphérique sur la santé, et malgré le caractère un peu alarmiste de cet article sur les neurotoxiques présents dans l'environnement, il nous a semblé intéressant d'en rendre compte sous forme d'un Faits et Chiffres afin d'en informer nos lecteurs. <

De nos jours, un enfant sur six aurait un handicap, touchant principalement le système nerveux. C'est du moins ce qu'estiment Philippe Grandjean et Philip Landrigan dans leur article qui dénombre 202 substances chimiques industrielles présentes dans l'environnement, susceptibles d'être neurotoxiques et qui pourraient donc être à l'origine de ces NDD (neurodevelopmental disorders) [1-3]. Ces NDD vont du simple défaut d'attention à des troubles beaucoup plus graves: hyperactivité, THDA (trouble d'hyperactivité avec déficit de l'attention), difficulté d'apprentissage, retard mental sévère, autisme. L'éventuelle relation de cause à effet entre les produits industriels neurotoxiques et ces NDD a été relativisée par la suite dans le Lancet par deux auteurs, l'un regrettant que la revue ait pris en compte une période

\section{Neurotoxicité des produits industriels et développement cérébral}

\section{Dominique Labie}

(Dominique Labie

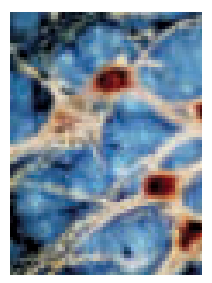

Département de génétique, développement et pathologie moléculaire, Institut Cochin,

24, rue du Faubourg Saint-Jacques,

75014 Paris, France.

où la surveillance était moins labie@cochin.inserm.fr

stricte [4], l'autre mettant en cause la définition des auteurs de la barrière hématoencéphalique [5]. II n'en reste pas moins que ces NDD sont une charge importante pour les familles et la société, que la responsabilité des neurotoxiques environnementaux doit être soigneusement recherchée et que tous les efforts doivent être entrepris pour les supprimer ou éviter qu'ils soient en contact avec des enfants ou des femmes enceintes.

\section{Le cerveau en développement est vulnérable}

Les enfants ne sont pas des petits adultes et le cerveau en développement est très sensible aux agressions toxiques. Or, le développement $d u$ cerveau commence tôt dans la vie fœtale et s'étend sur de nombreuses années. Au cours des neuf mois de la vie intra-utérine, le cerveau se développe à partir de cellules de l'ectoderme dorsal selon un schéma très rigoureux, qui permet les interconnexions des cellules entre elles. La chronologie très précise des migrations cellulaires définit des fenêtres de susceptibilité pour l'interférence d'un toxique, ne laissant pratiquement pas de possibilité d'une réparation ultérieure [6]. La barrière placentaire, en effet, laisse passer les métaux, et la barrière hématoencéphalique ne serait par ailleurs encore qu'incomplètement formée jusqu'à 6 mois. II faut aussi compter avec l'intervention d'un autre facteur, une absorption supérieure à celle de l'adulte et un moindre pouvoir de détoxification. Les substances lipophiles ou halogénées s'accumulent dans le tissu adipeux de la mère, passent dans le lait maternel, et peuvent se trouver 100 fois plus concentrées chez l'enfant [7]. Les neurotoxiques agissent sur l'ensemble des processus de développement: prolifération, migration, différenciation, synaptogenèse, gliogenèse, myélinisation, apoptose et signalisation et cette vulnérabilité se prolonge jusqu'à l'adolescence. Selon les auteurs, la nocivité des polluants de l'environnement s'exerce selon un continuum dont l'expression est dose-dépendante (Figure 1). Ce n'est qu'à partir 


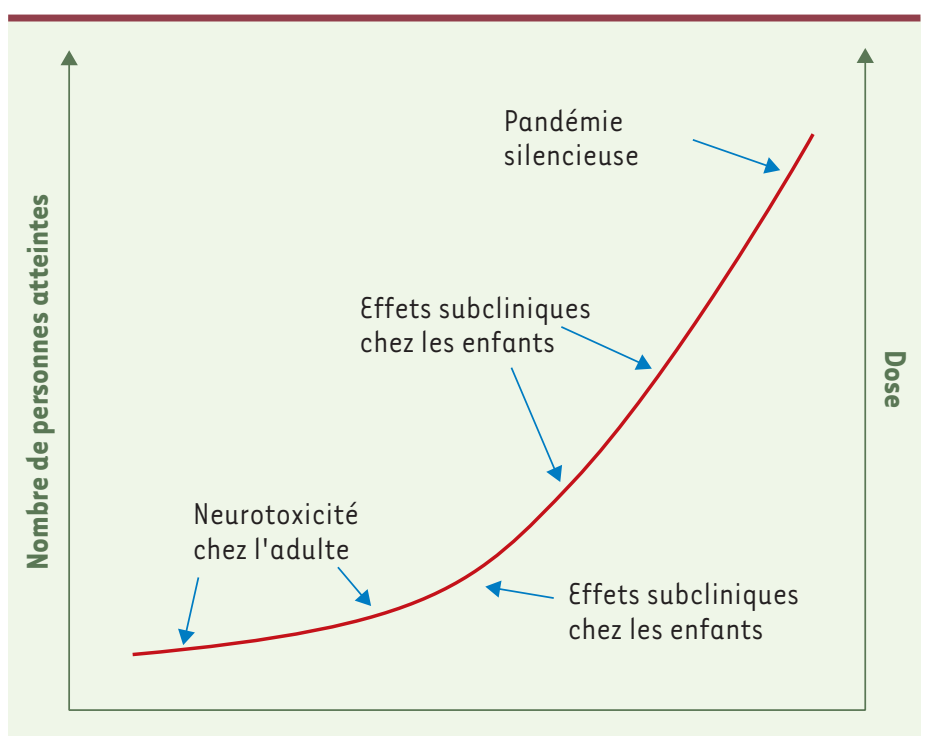

Figure 1. Action des toxiques les mieux documentés. Plomb, méthylmercure, biphénylpolychlorés, sans doute aussi l'arsenic et le toluène. De fortes doses ont déterminé des accidents neurotoxiques chez l'adulte. Les études épidémiologiques ont prouvé qu'à des doses plus faibles les enfants étaient intoxiqués. II existe une relation inverse entre le nombre de sujets affectés et les doses du produit chimique (d'après [1]).

de constatations cliniques faites chez l'adulte, puis d'études en expérimentation animale que la présence chez l'enfant de troubles intellectuels ou du comportement a été mise en évidence. L'existence d'une neurotoxicité n'a initialement été décelée qu'en présence de troubles fonctionnels évidents, et seules des recherches ultérieures ont décelé des désordres infracliniques ou latents, confirmés ultérieurement par des études épidémiologiques prospectives [8].

II faut en outre garder à l'esprit que 62000 produits chimiques étaient déjà enregistrés aux États-Unis avant que ne soit signé le Toxic Substances Control Act en 1977. De même en Europe, parmi 100000 produits industriels recensés dans la CE en 1981 [9], il en reste encore un grand nombre dont on ignore la toxicité.

\section{Les produits neurotoxiques}

Comment les répertorier? Les études chez l'animal confortent l'idée qu'une large gamme de molécules - qui ne sont pas nocives chez l'adulte - peuvent entraîner des troubles neurodéveloppementaux. Ceux-ci n'apparaissent que lorsque l'animal atteint sa maturité. Les examens toxicologiques de routine s'avèrent donc souvent inadéquats, et chez l'homme, ils doivent être complétés par des données cliniques et épidémiologiques. L'identification reste de ce fait incomplète. Parmi les 202 éléments recensés, les agents les mieux caractérisés sont les métaux, les solvants organiques et les pesticides, en particulier les composés organophosphorés, ainsi que de nouveaux produits, tels que les ignifugeants bromés. Dans la revue n'ont pas été abordés les consommables, dont l'alcool - qui a par ailleurs fait l'objet récemment d'un intéressant travail sur les conséquences de son absorption massive sur la mortalité en Russie [10].

La liste complète des produits nocifs est donnée dans la revue. Nous nous contenterons ici de mentionner les neurotoxiques avérés.

\section{Le plomb}

Le plomb est un métal présent partout dans notre quotidien. II y a près d'un siècle que des cas d'intoxication aigue par le plomb avaient été signalés en Australie chez des enfants qui, en jouant, avaient ingéré des peintures au plomb. La description de séquelles à long terme remonte aux années 1940. Malgré les mises en garde des pédiatres qui constataient la fréquence de déficits infra cliniques, le plomb est cependant resté largement utilisé (essence, peinture, céramiques) [11]. Entre-temps, les évidences se sont accumulées, démontrant l'importance des troubles, aboutissant à la suppression du plomb dans les carburants et à d'autres réglementations [12]. Ajoutons qu'en France, la découverte de cas de saturnisme, liés à la présence de fortes teneurs en plomb dans l'eau destinée à la consommation humaine dans les Vosges au début des années 1980, a conduit les pouvoirs publics à prendre des mesures afin de limiter ce risque. Conjointement, les ministères chargés de la santé, du logement et de l'écologie et tous les acteurs concernés se sont engagés dans une politique de réduction du plomb dans l'eau. Mais en 2005, Médecins du Monde rapportait dans son bilan d'activité la détection de 186 enfants dans les Hauts-de-Seine avec une plombémie élevée : $18 \%$ > à $100 \mu \mathrm{g} / \mathrm{l}$, et $11 \%$ comprise entre 50 et $100 \mu \mathrm{g} / \mathrm{l}$.

La Commission européenne, se fondant sur les recommandations de l'Organisation mondiale de la Santé, a décidé d'abaisser les seuils en vigueur. À partir du 25 décembre 2003, la valeur limite de $50 \mu \mathrm{g} / \mathrm{I}$ fut abaissée à $25 \mu \mathrm{g} / \mathrm{l}$, puis sera limitée à $10 \mu \mathrm{g} / \mathrm{l}$ à partir du 25 décembre 2013.

\section{Le méthylmercure}

Les premières observations ont été faites à Minamata au Japon dans les années 1960 à l'occasion d'une épidémie de cécité et de retard mental chez des enfants dont les mères avaient consommé des eaux contaminées. II a fallu plusieurs années pour qu'on identifie l'usine de plastique à l'origine de cette contamination. Les adultes étaient peu, ou pas atteints, alors que les enfants présentaient des troubles graves du développement neurologique. Des travaux récents se sont focalisés sur l'effet qu'auraient avant la naissance de faibles concentrations 
de méthylmercure dans des populations qui consomment en abondance poisson et fruits de mer, selon le degré de contamination de l'eau. De tels travaux menés en Nouvelle-Zélande, et aux îles Féroé, ont noté des troubles de mémoire. Une étude très complète a aussi été réalisée en Guyane Française avec analyse comparative de populations exposées (en raison des activités d'orpaillage) et non exposées, comportant des dosages de méthylmercure dans les cheveux ainsi que des examens neuropsychologiques et des tests de développement comportemental [13]. Un autre travail important, mené aux Seychelles sur 779 couples mère-enfant, n'a cependant pas confirmé l'hypothèse d'une action neurotoxique prénatale par simple consommation de poisson [14]. Un contrôle strict de l'usage du méthylmercure, son élimination dans les hôpitaux et les incinérateurs sont demandés dans le programme environnemental de 2002 des Nations-Unies [15].

\section{Les diphénylpolychlorés}

Ces produits (PCB) ont été utilisés comme isolants électriques, et des contaminations industrielles ont été décrites dans des pays asiatiques, Japon et Taiwan. Les études épidémiologiques mettent en évidence un déficit infra clinique du développement: hyperactivité, troubles de la mémoire et de l'attention, $\mathrm{Ql}$ total et verbal diminué. Les troubles s'observent dans l'enfance et la préadolescence chez des sujets ayant été exposés pendant la vie fœtale [16]. Un mécanisme possible serait une interférence avec la fonction thyrö̈dienne maternelle [17]. La fabrication en a été interdite depuis 1977.

\section{Les solvants}

Les intoxications aigues, souvent professionnelles, sont bien connues, elles sont présentes dans les sociétés industrialisées et responsables de nombreuses intoxications, touchant le système nerveux central ou périphérique [18]. L'un des solvants les plus étudiés fut le toluène, très utilisé jadis en laboratoire. Les données sont moins précises concernant d'autres solvants auxquels serait exposée la mère pendant sa grossesse.

\section{Les pesticides}

On en connaît plus de 600 : insecticides, fongicides ou raticides. Un de leurs mécanismes d'action serait une inhibition de la cholinestérase par les organophosphates. Les premières descriptions ont été faites au Mexique, en comparant des cohortes d'enfants qui avaient été exposés ou non à des pesticides [19]. Des résultats analogues ont été retrouvés aux États-Unis, des enfants de régions agricoles s'avérant moins performants que ceux des zones urbaines. On a aussi décrit en Équateur un développement hypotrophique chez des enfants intoxiqués. Les séquelles neurologiques d'une exposition prénatale à certains organophosphates ont été démontrées [20]. Des modifications morphologiques ont aussi été signalées: l'exposition prénatale au chlorpyrifos est associée à une baisse des scores de développement à 36 mois ainsi qu'à un risque de THDA augmenté [21].

\section{Le manganèse}

Le rôle du manganèse dans des maladies professionnelles est connu, entraînant des troubles neurodégénératifs voisins de la maladie de Parkinson. Son intervention dans le développement neurologique s'est posée du fait que, comme l'arsenic, le manganèse existe dans des nappes phréatiques utilisées pour l'eau de boisson (Bangladesh) et dans le lait de soja. Une étude épidémiologique menée sur 247 couples mère/enfant a montré une relation entre le taux du manganèse dans le sang de cordon et des troubles mineurs du développement à l'âge de trois ans [22]. Pire, la présence simultanée de manganèse, d'arsenic et de cadmium a été observée dans les cheveux d'enfants vivant à proximité d'une décharge [23]. Le MMT (méthylcyclopentadiényl manganèse tricarbonyle) est un additif qui améliore l'indice d'octane dans l'essence sans plomb. On l'utilise au Canada depuis 1977 et, plus précisément, depuis qu'on n'ajoute plus à l'essence d'additifs de plomb. Mais ce manganèse méthylé présent dans l'essence et dans les émissions de gaz d'échappement augmente la quantité de Mn dans l'air, le sol et la poussière.

\section{Les fluorures, le perchlorate}

Leur neurotoxicité en est encore mal documentée. Celle des fluorures n'a été démontrée que chez des animaux de laboratoire, et les perchlorates ne sont pas toxiques chez l'adulte. Ils sont cependant fréquents dans l'eau, et pourraient agir sur la fonction thyroïdienne. Il est sûr que la liste des toxiques industriels possibles est loin d'être exhaustive.

\section{Les produits émergents}

Ajoutés à de nombreux produits pour inhiber l'allumage et la propagation des flammes, les polybromodiphényléthers (PBDE), ces ignifugeants bromés (on en compte plus de 75 variétés) sont un nouveau sujet de préoccupation. Leurs concentrations dans l'environnement et dans l'organisme humain, quoique faibles, sont en hausse en Amérique du Nord et plus élevées qu'en Europe, où ils sont interdits (Figure 2). Neurotoxiques pendant une période critique du développement cérébral chez la souris [24], ils n'ont pas été documentés chez l'homme.

\section{Comment évaluer la neurotoxicité potentielle des produits chimiques?}

Dans tous les cas où la neurotoxicité est avérée, le scénario a toujours été le même. Elle est découverte chez l'adulte, généralement en tant que maladie professionnelle, ou chez l'enfant du fait d'un accident aigu d'empoisonnement. Les études épidémiologiques recherchent ensuite un déficit du développement neu- 


\section{Niveaux de PBDE chez l'humain et l'animal}

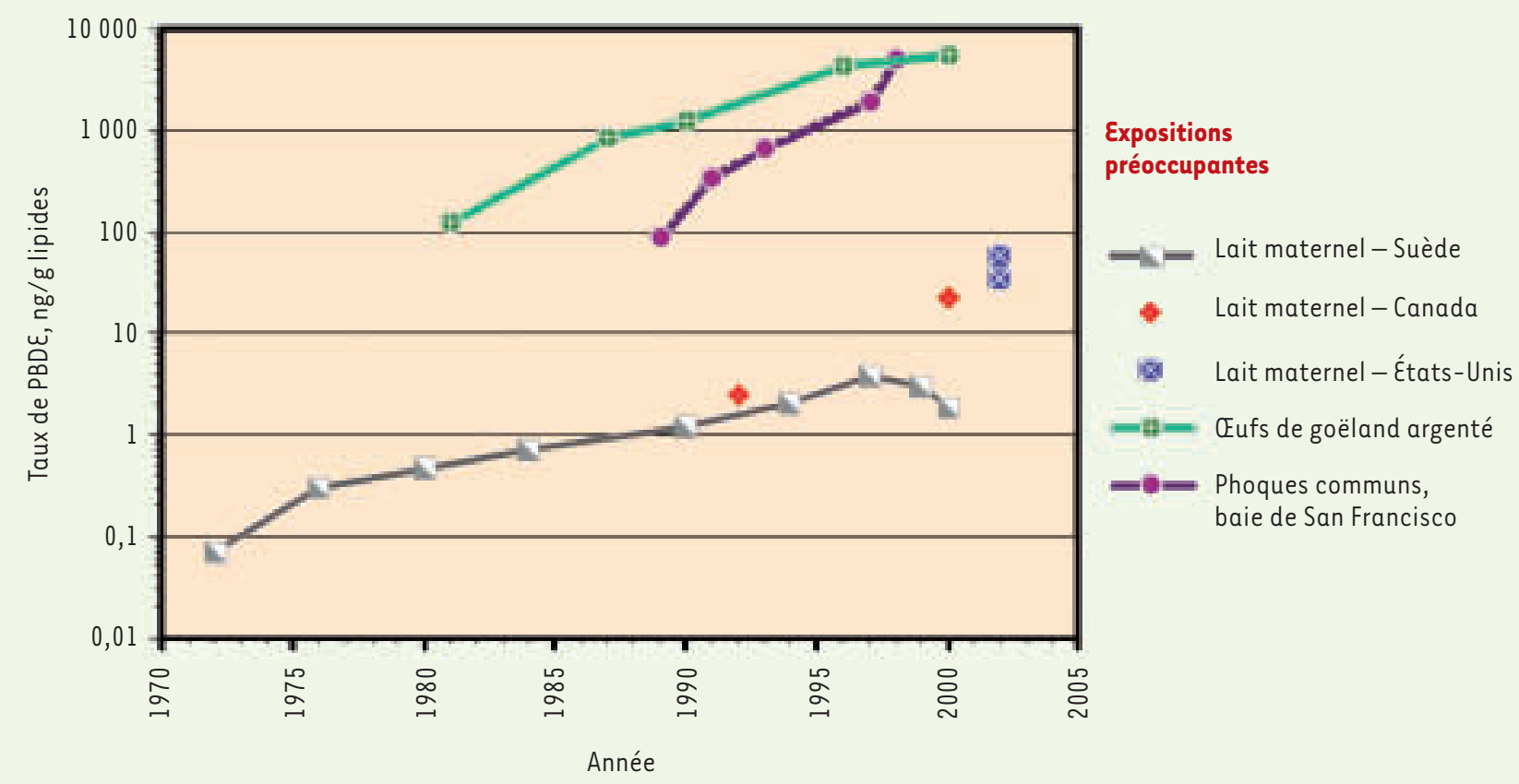

Figure 2. Taux de PBDE (polybromodiphényléthers) dans le lait maternel aux États-Unis et au Canada (en 1992, 2000 et 2002 ), et de 1970 à 2002 en Suède. Les concentrations ont diminué peu après l'adoption de mesures. En outre sont mentionnées les concentrations dans deux espèces de poissons (d'après le cours de Ted Schettle - mai 2007 - sur les risques environnementaux au développement du cerveau, Moncton, Nouveau Brunswick, Canada).

rologique, quelquefois infraclinique, et l'attribuent à l'exposition au toxique, le plus souvent pendant la période prénatale. Hormis les QI nettement inférieurs à la normale, qu'en est-il de l'hyperactivité, des THADA, des troubles du comportement, des déficits de mémoire, des inadaptations sociales? Existe-t-il des effets retardés ou latents?

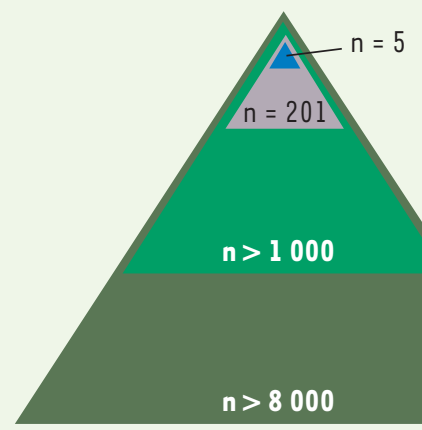

Produits toxiques pour le neurodéveloppement

Produits toxiques chez l'homme pour le neurodéveloppement

Figure 3. Proportions relatives des toxiques dont l'action sur le développement cérébral est documenté. Ceux que l'on sait toxiques, ceux dont la toxicité n'a été étudiée qu'expérimentalement, et, enfin, des dizaines de milliers de produits qui constituent l'univers de la chimie (d'après [1]).
Y a-t-il, par exemple, des cas de maladie de Parkinson dus à la destruction silencieuse de cellules dont le manque ne se révélerait que tardivement? Comment évaluer l'importance de ces handicaps et leur retentissement économique? Seule, une accumulation systématique de données pourra répondre à ces questions. À l'heure actuelle, si on compte sur les doigts d'une main les produits dont l'action sur le développement du cerveau a été démontrée, il en existe quelques dizaines reconnus comme toxiques, quelques centaines dont la toxicité a été étudiée expérimentalement, et des milliers sur lesquels on ne sait rien. Nos connaissances ne portent que sur la partie émergée de l'iceberg (Figure 3). À toutes ces questions, seule l'étude de grandes cohortes, avec des tests de DNT (developmental neurotoxicity tests) et des comparaisons internationales, pourra apporter une réponse. Dès 1995, I'OCDE (Organisation de coopération et de développement économiques) a commencé l'élaboration de directives sur les DNT avec :

- variations des dosages et du type d'exposition,

- choix des espèces animales compte tenu des propriétés du produit à étudier,

- étude chez les mères et les petits, évaluation de la fonction et de la structure neurale tout au long du cycle de vie, - analyses motrice, sensorielle, et comportementale plus complexes... 
Les connaissances actuelles justifient les réglementations concernant l'élimination des toxiques, comme le plomb ou le méthylmercure. Elles justifient aussi les précautions prescrites pendant la grossesse et en période d'allaitement et la multiplication des DNT. $\diamond$

\section{SUMMARY}

Developmental neurotoxicity of industrial chemicals

«A Silent Pandemic: Industrial Chemicals Are Impairing the Brain Development of Children Worldwide » Fetal and early childhood exposures to industrial chemicals in the environment can damage the developing brain and can lead to neurodevelopmental disorders (NDDs) - autism, attention deficit disorder (ADHD), and mental retardation. In a new review study, published in The Lancet, Philip Grandjean and Philip Landrigan from the Harvard School of Public Health systematically examined publicly available data on chemical toxicity in order to identify the industrial chemicals that are the most likely to damage the developing brain. The researchers found that 202 industrial chemicals have the capacity to damage the human brain, and they conclude that chemical pollution may have harmed the brains of millions of children worldwide. The authors conclude further that the toxic effects of industrial chemicals on children have generally been overlooked. In North Amercia, the commission for environmental cooperation, and in European Union the DEVNERTOX projects had reached to the same conclusions. We analyse this review and discuss these rather pessimistic conclusions.

\section{RÉFÉRENCES}

1. Grandjean P, Landrigan PJ. Developmental neurotoxicity of industrial chemicals. Lancet 2006; $368: 2167-78$.

2. Commission de coopération environnementale 2006. Les substances toxiques et la santé des enfants en Amérique du Nord. www.cec.org/files/PDF/JPAC/SR-05-02_fr.pdf

3. Toxic threats to the developing nervous system : in vivo and in vitro studies on the effects of mixture of neurotoxic substances potentially contaminating food. http://www.imm.ki.se/ devnertox/

4. Plunkett LM. Developmental neurotoxicity of industrials chemicals. Lancet $2007 ; 369: 821-2$.

5. Saunders NR, Dziegielewska KM. Developmental neurotoxicity of industrials chemicals. Lancet $2007 ; 369: 821-2$.

6. Rice $D$, Barone RD. Critical periods of vulnerability for the developing nervous system : evidence from humans and animal models. Environ Health Perspect $2000 ; 118: 511-33$.

7. Landrigan PJ, Schechter CB, Lipton JM, et al. Environmental pollutants and disease in American children : estimates of morbidity, mortality, and costs for lead poisoning, asthma, cancer, and developmental disabilities. Environ Health Perspect 2002 ; 110 : 721-8.
8. Ginsberg G, Hattis D, Sonawane B. Incorporating pharmacokinetic differences between children and adults in assessing children's risks to environmental toxicants. Toxicol Appl Phamacol $2004 ; 198$ : 164-83.

9. Commission of the European Communities: White paper. Strategy for a future chemical policy (COM 200188 final, 2001). http//europa.eu.int/ comm/environment/chemicals/pdf/0188_en.pdf (2005)

10. Leon DA, Saburova L, Tomkins $S$, et al. Hasardous alohol drinking and premature mortalitiy in Russia : a population based case-control study. Lancet 2007 ; $369: 2001-9$.

11. Landrigan PJ, Whitworth RH, Balow RW, et al. Neuropsychological dysfunction in children with chronic low level lead absorption. Lancet $1975 ;$ I: 708-12.

12. Lanphear BP, Hornung R,Khoury J, et al. Low-level environmental lead exposure and children's intellectual function: an international pooled anlysis. Environ Health Perspect 2005 ; 113 : 894-9.

13. Myers GJ, Davidson PW, Cox C, et al. Prenatal methylmercury exposure from ocean fish consumption in the Seychelles child development study. Lancet 2003 ; $361:$ 1686-92.

14. Cordier S, Garel M. Risques neurotoxiques chez l'enfant liés à I'exposition au méthylmercure en Guyane Française. www.invs.sante.fr/publications/ mercure_guyane/index.html

15. United Nations environment programme. Global mercury assessment. Geneva, Switzerland, 2002.

16. Jacobson JL, Jacobson SW. Intellectual impairment in children exposed to polychlorinated biphenyls in utero. N Engl J Med 1996 ; 335 : 783-9.

17. Takser L, Mergler D, Baldwin M, et al. Thyroid hormone in pregnancy in relation to environmental exposure to organochloride compounds and mercury. Environ Health Perspect 2005 ; 113 : 1039-45.

18. White RF, Proctor SP. Solvents and neurotoxicity. Lancet 1997 ; 349 : 1239-41.

19. Guillette A, Meza MM, Aquilar MG, et al. An anthropological approach to the evaluation of preschool children exposed to pesticides in Mexico. Environ Health Perspect 1998; 106 : 147-51

20. Ruckkart PZ, Kakolewski K, Bove FJ, Kaye WE. Long-term neurobehavioral and health effects of methyl parathion exposure in children in Mississipi and Ohio. Environ Health Perspect 2004 ; 112 : 46-51.

21. Landrigan PJ, Trasande L, Thorpe LE, et al. The National Children's Study a 21-year prospective study of 100,000 American children. Pediatrics $2006 ; 118: 2173-86$.

22. Takser L, Mergler D, Hellier G, et al. Manganese, monoamine metabolite levels at birth, and child psychomotor development. NeuroToxicol 2003 2466: 7-74.

23. Wright R0, Amarasiriwardena C, Woolf AD, et al. Neuropsychological correlates of hair arsenic, manganese, and cadmium levels in school-age children residing near a hazardous waste site. NeuroToxicol 2006; 27 : 210-6.

24. Viberg H, Fredriksson A, Eriksson P. Neonatal exposure to polybrominated diphenyl ether (PBDE 153) disrupts spontaneous behaviour, impairs learning and memory, and decreases hippocampal cholinergic receptors in adult mice. Toxicol Appl Pharmacol $2003 ; 192: 95-106$.

\section{TIRÉS À PART}

D. Labie

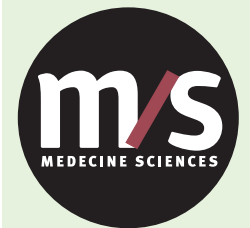

Tarifs d'abonnement M/S - 2007

Abonnez-vous

à Médecine/Sciences
> Depuis 20 ans, grâce à $m / s$, vous vivez en direct les progrès des sciences biologiques et médicales

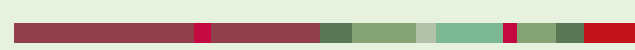

Bulletin d'abonnement page 790 dans ce numéro de $\mathrm{m} / \mathrm{s}$
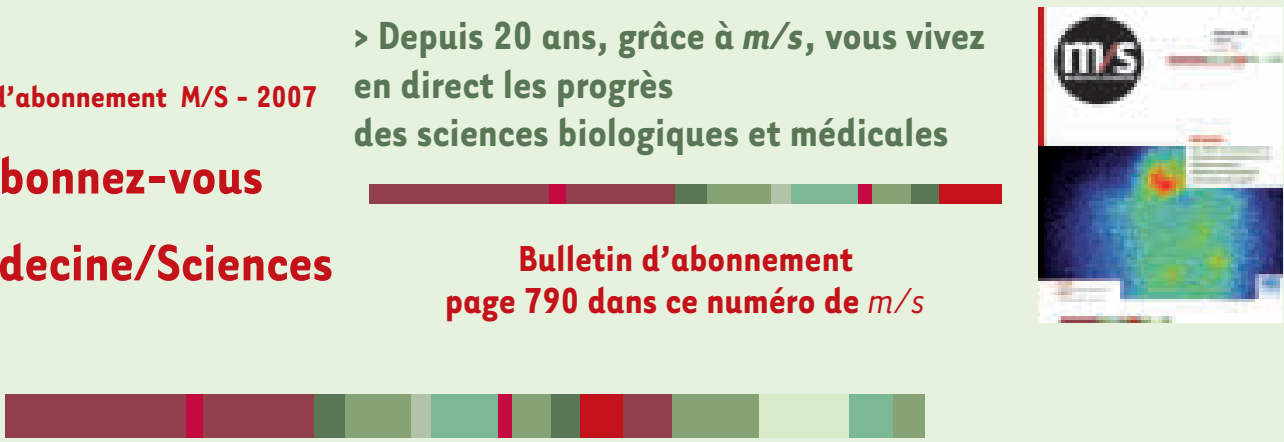